\title{
Cervical Spinal Cord Ependymal Tumor
}

National Cancer Institute

\section{Source}

National Cancer Institute. Cervical Spinal Cord Ependymal Tumor. NCI Thesaurus. Code C131601.

An ependymal tumor arising from the cervical region of spinal cord. 